\title{
Increasing posterior condyle cut for high-flex knee prosthesis may injure popliteus tendon origin: a cadaveric study
}

\section{Rit Apinyankul ${ }^{1}$, Surachai Sae-Jung ${ }^{1}$ and Ong-art Phruetthiphat ${ }^{2 *}$ \\ ${ }^{1}$ Department of Orthopaedics, Faculty of Medicine, Khon Kaen University, Khon Kaen, Thailand ${ }^{2}$ Orthopaedics Department, Phramongkutklao Hospital, Bangkok, Thailand}

*Correspondingauthor: Ong-artPhruetthiphat, Orthopaedics Department, Phramongkutklao Hospital, Bangkok, Thailand, 10400, Tel: +66814085470; E-mail: ongart-phr1@hotmail.com

\section{Research Article \\ Volume 4 Issue 1}

Received Date: February 20, 2020

Published Date: March 04, 2020

DOI: $10.23880 /$ jobd-16000193

\section{Abstract}

Background: High-flex total knee prosthesis designs were proposed to improve flexion in total knee replacement (TKA). One of high-flex features is increasing posterior condyle cut which put popliteal tendon in higher risk of injury and may result in gap changes.

Methods: Thirty-six popliteal origin sites from eighteen fresh cadavers were measured distances between the posterior rim of popliteal tendon origin and posterior border of the lateral femoral condyle (distance A) using digital "Vernier caliper". The mean distances were compared to posterior condyle thickness of different prosthesis designs.

Results: The mean of distance $A$ on the right knee was $9.59 \pm 1.66 \mathrm{~mm}(6.03-12.70)$ while the mean of distance $A$ on the left knee was $9.13 \pm 1.78 \mathrm{~mm}$ (5.80-11.07). Posterior condyle thickness of the femoral prostheses varies upon their design and size from 7.4 to $10 \mathrm{~mm}$ for standard model and from 8.2 to $12.5 \mathrm{~mm}$ for high-flex design. Possibilities of popliteal tendon injury during posterior condyle bone cut were $16.7 \%$ to $66.7 \%$ for standard model and $27.8 \%$ to $97.2 \%$ for the high-flex design.

Conclusion: High-flex TKA prosthesis with thicker posterior condyle relates to higher possibility of popliteal tendon origin injury compared to standard one.

Keywords: High-flex; TKA; Popliteal tendon injury; Asian population; Posterior Condyle Cut; Cadaveric study

\section{Introduction}

Total knee arthroplasty (TKA) surgery becomes standard treatment for advance-staged knee osteoarthritis for many decades. However, most of conventional design total knee prosthesis provide knee flexion not more than 115 degrees [1-4]. High-flex knee prosthesis design was proposed to improve knee flexion and provide a range of motion more than the conventional one by changing several features: reduced posterior femoral condylar radii with thickened posterior femoral condyles, reshaping the posterior contour of polyethylene liner and more posterior condyle bone cut to reduce impingement during deep flexion.
Popliteal tendon provides both static and dynamic stabilities in the normal knee [5-7]. Previous studies demonstrated gap changes in PS-TKA cadaveric surgery with popliteal tendon rupture [8] and the popliteal tendon resection resulted in destabilizing of lateral and medial aspects of the knee [9]. Additionally, we observed some of popliteal tendon injuries during posterior condyle cutting step of high-flex TKA surgery. To our knowledge, no study revealed relationships between popliteal tendon injury and posterior condyle cutting. The purpose of our study was to investigate the posterior femoral condyle to find out the risk of popliteal tendon injury associated with high-flex and standard model TKA. 


\section{Materials and Methods}

The study protocol was reviewed by the institute's Ethics Committee for Human Research, and deemed exempt from the institutional review board oversight because the study met the criteria of the Exemption Determination Regulations (research involving the collection of study of bone, the body of persons who donate to the hospital and subjects cannot be identified directly or through identifiers linked to the subjects).
The study was designed as cadaveric research to determine and measure popliteal tendon origin footprints accurately. Eighteen fresh cadavers were dissected to reveal thirty-six popliteal tendon origin sites and posterior border of the lateral femoral condyle. The distance between the posterior rim of popliteal tendon origin and posterior border of the lateral femoral condyle was measured using a digital "Vernier caliper" (Distance A, Figure 2). The distance A was measured three times and the average of them were calculated.
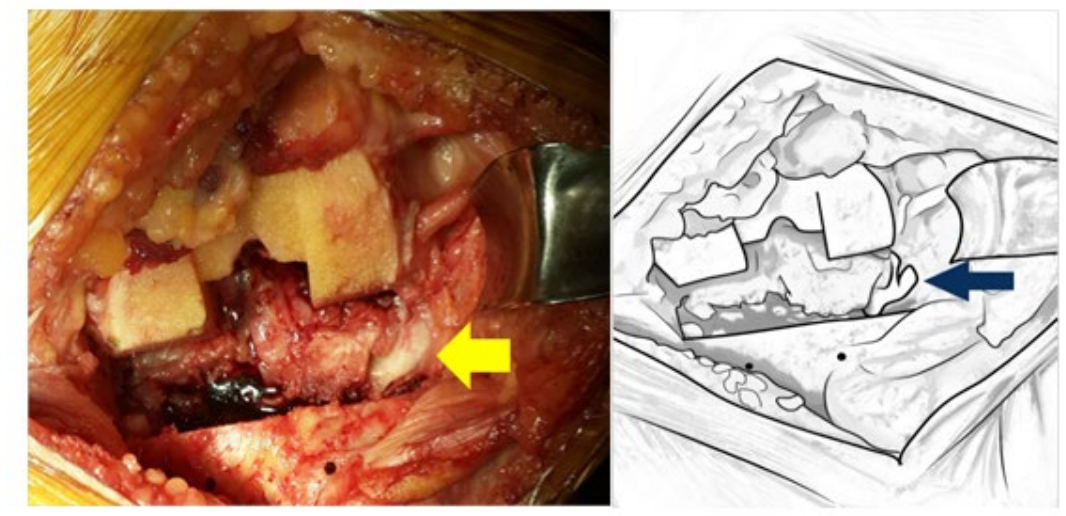

Figure 1: Popliteal tendon injury ( yellow arrow) during knee flexion after posterior condylar bone cut in high-flex prothesis design.

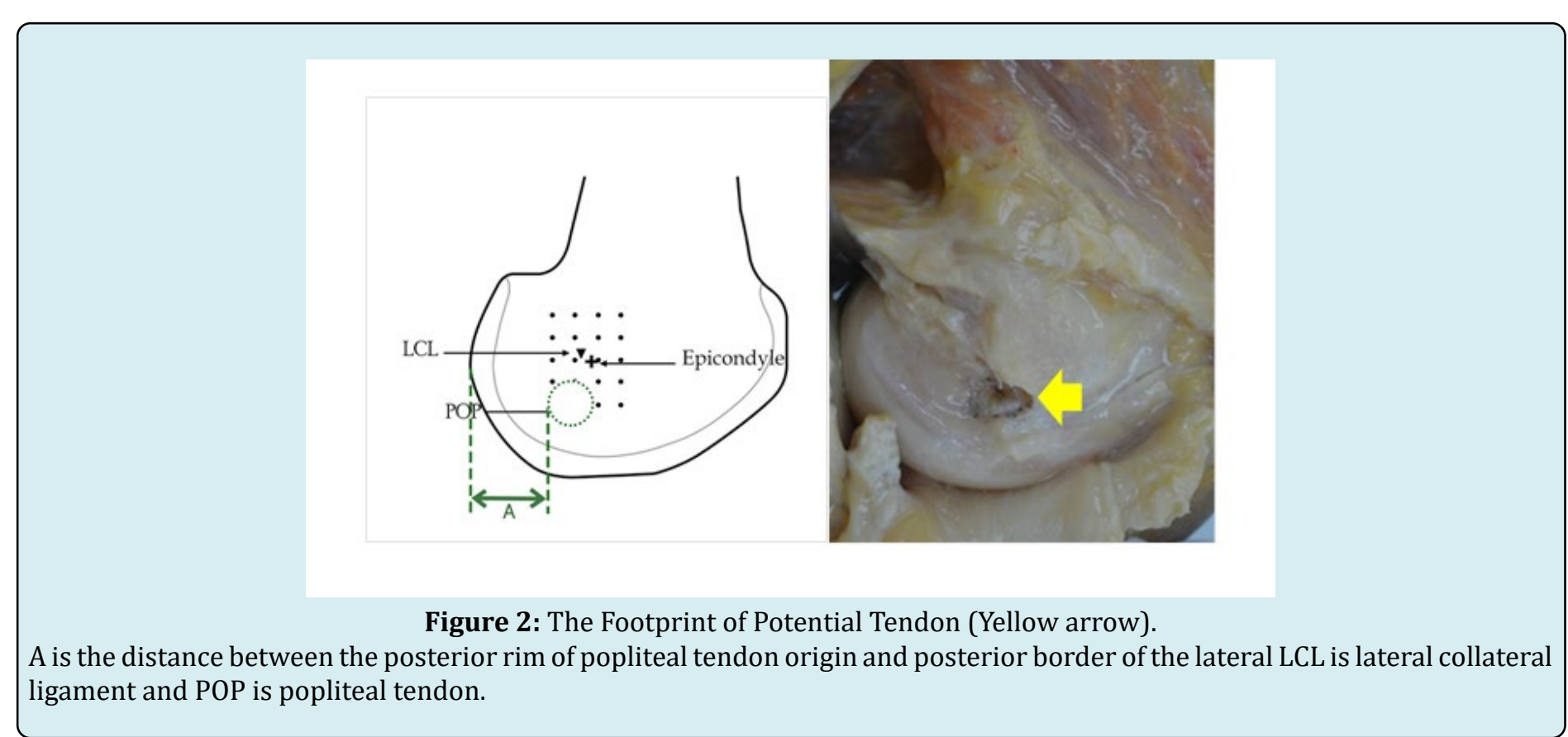




\section{Data Analysis}

The data was collected and calculated using mean and standard deviation values including minimum and maximum to analyze data of distance $\mathrm{A}$.

\section{Results}

The study revealed a mean of distance A $9.38 \mathrm{~mm}$ (SD $1.68 \mathrm{~mm}$ ). Details for each cadaver were shown in table 1. The mean of distance $A$ on the right knee was $9.59 \pm 1.66 \mathrm{~mm}$ (6.03-12.70) while the mean of distance A on the left knee was $9.13 \pm 1.78 \mathrm{~mm}(5.80-11.07)$.

The possibilities of popliteus injury when using a standard prosthesis and high flex prosthesis were demonstrated in table 2, 3 respectively. The posterior condyle thickness of the femoral prostheses varies upon their design and size from 7.4 to $10 \mathrm{~mm}$ for standard model and from 8.2 to $12.5 \mathrm{~mm}$ for high-flex design. If the patient's distance of
A is shorter than the posterior condyle thickness, there will be a risk for popliteus origin injury during posterior condyle cut. As a result, the possibilities of popliteal tendon injury during posterior condyle bone cut were $16.7 \%$ to $66.7 \%$ for standard model and between $27.8 \%$ to $97.2 \%$ for the highflex design.

The possibly popliteal injury was variable depending on the thickness of posterior condyle of each prosthesis and the distance between posterior rim of popliteal tendon origin and posterior border of the lateral femoral condyle as shown in tables 2 and 3.

Distance $A$ is the distance between the posterior rim of popliteal tendon origin and posterior border of the lateral femoral condyle. $A_{R}$ and $A_{L}$ are the distance $A$ of right and left knee, respectively. Average $A_{R}$ was $9.59 \pm 1.66 \mathrm{~mm}(6.03-$ $12.70)$ and Average $A_{L}$ was $9.13 \pm 1.78 \mathrm{~mm}(5.80-11.07)$. The average of all cadavers was $9.36 \pm 1.71 \mathrm{~mm}(5.80-12.70)$.

\begin{tabular}{|c|c|c|c|}
\hline Cadaver & Gender & AR(mm) & AL(mm) \\
\hline 1 & Male & 10.57 & 10.73 \\
\hline 2 & Male & 11.27 & 10.43 \\
\hline 3 & Male & 10.73 & 10.47 \\
\hline 4 & Male & 9.9 & 11.07 \\
\hline 5 & Male & 9.6 & 9.47 \\
\hline 6 & Male & 10.33 & 10.13 \\
\hline 7 & Male & 10.8 & 10.17 \\
\hline 8 & Male & 10.27 & 9.43 \\
\hline 9 & Male & 10.47 & 6.37 \\
\hline 10 & Male & 6.73 & 6.3 \\
\hline 11 & Female & 8.17 & 10.7 \\
\hline 12 & Male & 10.07 & 5.8 \\
\hline 13 & Male & 6.03 & 7.7 \\
\hline 14 & Male & 12.7 & 7.3 \\
\hline 15 & Male & 7.73 & 11.07 \\
\hline 16 & Male & 9.97 & 7.63 \\
\hline 17 & Male & 8.2 & 9.93 \\
\hline 18 & Male & 9.03 & \\
\hline $1: T h$ & & & \\
\hline
\end{tabular}

Table 1: The measurement of distance between the posterior rim of popliteal tendon origin and posterior border of the lateral femoral condyle. 
Journal of Orthopedics \& Bone Disorders

\begin{tabular}{|c|c|c|c|}
\hline Prosthetic Design & $\begin{array}{l}\text { Thickness of posterior condyle } \\
\text { (millimeters) }\end{array}$ & $\begin{array}{c}\text { The average thickness of } \\
\text { distance A (Cadavers) }\end{array}$ & $\begin{array}{l}\text { Possibly popliteal tendon } \\
\text { injury in clinical practice }\end{array}$ \\
\hline Anthem & \multirow{2}{*}{9.5} & \multirow{23}{*}{$9.36 \pm 1.71 \mathrm{~mm}(5.8-12.7 \mathrm{~mm})$} & \multirow{2}{*}{$38.90 \%$} \\
\hline PS and CR & & & \\
\hline Legion & \multirow{2}{*}{9.5} & & \multirow{2}{*}{$38.90 \%$} \\
\hline PS, CR and CK & & & \\
\hline Journey II & \multirow{2}{*}{7.4 (posterior lateral) } & & \multirow{2}{*}{$16.70 \%$} \\
\hline PS design & & & \\
\hline Sigma & \multirow{2}{*}{7.6 to 8.5} & & \multirow{2}{*}{$16.7 \%$ to $30.6 \%$} \\
\hline PS & & & \\
\hline Attune & \multirow{2}{*}{9} & & $3060 \%$ \\
\hline PS and CR & & & $30.00 \%$ \\
\hline Nexgen & \multirow{2}{*}{10.4} & & \multirow{2}{*}{$66.70 \%$} \\
\hline PS & & & \\
\hline Persona & \multirow{2}{*}{9 to 10} & & \multirow{2}{*}{$30.6 \%$ to $52.8 \%$} \\
\hline PS & & & \\
\hline $\mathrm{CR}$ & 8 to 9 & & $25.0 \%$ to $30.6 \%$ \\
\hline Triathlon & \multirow{2}{*}{8.5} & & \multirow{2}{*}{$30.60 \%$} \\
\hline PS and CR & & & \\
\hline Vanguard & \multirow{2}{*}{9} & & \multirow{2}{*}{$30.60 \%$} \\
\hline PS and CR & & & \\
\hline Columbus & \multirow{2}{*}{8} & & \multirow{2}{*}{$25.00 \%$} \\
\hline PS & & & \\
\hline Vega & \multirow{2}{*}{10} & & \multirow{2}{*}{$52.80 \%$} \\
\hline PS & & & \\
\hline
\end{tabular}

Table 2: Comparison of Posterior Condyle Thickness of Prosthetic Design (Standard Model), Distance A and Possibility of Popliteal Tendon Injury.

\begin{tabular}{|c|c|c|c|}
\hline Prosthetic Design & $\begin{array}{l}\text { Thickness of posterior } \\
\text { condyle (millimeters) }\end{array}$ & $\begin{array}{l}\text { The average thickness } \\
\text { of distance A (Cadav- } \\
\text { ers) }\end{array}$ & $\begin{array}{l}\text { Possibly popliteal tendon } \\
\text { injury in clinical practice }\end{array}$ \\
\hline Anthem & \multirow{2}{*}{11.5} & \multirow{9}{*}{$\begin{array}{c}9.36 \pm 1.71 \mathrm{~mm}(5.8-12.7 \\
\mathrm{mm})\end{array}$} & \multirow{2}{*}{$97.20 \%$} \\
\hline PS and $C R$ & & & \\
\hline Legion & \multirow{2}{*}{9.4 (posterior lateral) } & & \\
\hline PS, CR and CK & & & \multirow{3}{*}{$33.40 \%$} \\
\hline & & & \\
\hline Sigma & \multirow{2}{*}{8.2 to 12.5} & & \\
\hline RP-F & & & \multirow{2}{*}{$27.8 \%$ to $97.2 \%$} \\
\hline Nexgen & \multirow{2}{*}{12.5} & & \\
\hline PS (High flex) & & & $97.20 \%$ \\
\hline
\end{tabular}

Table 3: Comparison of Posterior Condyle Thickness of Prosthetic Design (High-Flex Model), Distance A and Possibility of Popliteal Tendon Injury. 


\section{Journal of Orthopedics \& Bone Disorders}

\section{Discussion}

Popliteal tendon provides both static and dynamic stabilities in the normal knee [5-7]. Previous studies demonstrated gap changes in PS-TKA cadaveric surgery with popliteal tendon rupture [8]. Additionally, popliteal tendon resection resulted in destabilizing of lateral and medial aspects of the knee [9]. During posterior condyle cutting step of high-flex TKA, we observed some of popliteal tendon injuries during high-flex TKA surgery. To our knowledge, no study revealed relationships between popliteal tendon injury and posterior condyle cutting. The purpose of our study was to investigate the posterior femoral condyle to find out the risk of popliteal tendon injury associated with high-flex TKA.

This study showed that an average of distance of $A$ was $9.38 \pm 1.68 \mathrm{~mm}$ (range $5.80-12.70 \mathrm{~mm}$ ) and they represent safe zone for posterior condyle cut without popliteus origin injury (Table 1). Additionally, the average distance A on the right side was larger than the left side (9.59 $9.13 \mathrm{~mm}$ ). It is possible that the injury to popliteal tendon when performing a standard designed or high flex designed TKA may be higher rate on left knee than right knee.

The results showed possibilities of popliteus injury when using a standard prosthesis (Table 2) and a high flex prosthesis (Table 3). The posterior condyle thickness of the femoral prostheses varies upon their design and size from 7.4 to $10 \mathrm{~mm}$ for standard model and from 8.2 to 12.5 $\mathrm{mm}$ for high-flex design. Our result demonstrated that the possibilities of popliteal tendon injury during posterior condyle bone cut were $16.7 \%$ to $66.7 \%$ for standard model and between $27.8 \%$ to $97.2 \%$ for the high-flex design. This is useful for counseling purpose for patients and their families who undergo TKA, especially with high-flex design.

Study limitation: A cadaveric study came from Thai people may represent only for Asian population who has smaller bone and smaller popliteal tendon. This may imply that the distance A may be shorter than another population (Caucasian, Hispanic, etc). Therefore, the possible rate of popliteal tendon injury when perform high-flex design TKA may be lower in Non-Asian population compared to Asian population.

\section{Conclusion}

Thai patients representing an Asian population have variety in popliteal tendon origin footprints and have a chance of its origin site injury during high-flex TKA surgery. The thicker prosthetic posterior condyle, the higher chance of popliteal origin site injury. Additionally, High-flex TKA prosthesis with thicker posterior condyle relates to higher possibility of popliteal tendon origin injury compared to standard one.

\section{Acknowledgments}

We would like to thank professor Sukit Saengnipanthkul for assistance in creating idea.

\section{References}

1. Murphy M, Journeaux S, Russell T (2009) High-flexion total knee arthroplasty: a systematic review. Int Orthop 33(4): 887-893.

2. Rowe PJ, Myles CM, Walker C, Nutton R (2000) Knee joint kinematics in gait and other functional activities measured using flexible electro goniometry: how much knee motion is sufficient for normal daily life? Gait Posture 12(2): 143-155.

3. Mulholland SJ, Wyss UP (2001) Activities of daily living in non-Western cultures: range of motion requirements for hip and knee joint implants. Int J Rehabil Res 24(3): 191-198.

4. Rousseau MA, Lazennec JY, Catonné Y (2008) Early mechanical failure in total knee arthroplasty. Int Orthop 32(1): 53-56.

5. Tria AJ Jr, Johnson CD, Zawadsky JP (1989) The popliteus tendon. J Bone Joint Surg Am 71(5): 714-716.

6. Pasque C, Noyes FR, Gibbons M, Levy M, Grood E (2003) The role of the popliteofibular ligament and the tendon of popliteus in providing stability in the human knee. J Bone Joint Surg Br 85(2): 292-298.

7. LaPrade RF, Woznicka JK, Stellmaker MP, Wijdicks CA (2010) Analysis of the static function of the popliteus tendon and evaluation of an anatomic reconstruction: the "fifth ligament" of the knee. Am J Sports Med 38(3): 543-549.

8. Tantavisut S, Tanavalee A, Ngarmukos S, Limtrakul A, Wilairatana V, et al. (2012) Gap changes after popliteustendon resection in PS-TKA: a cadaveric study in Thai female knees. Knee 19(5): 597-600.

9. Cottino U, Bruzzone M, Rosso F, Dettoni F, Bonasia DE, et al. (2015) The role of the popliteus tendon in total knee arthroplasty: a cadaveric study. Joints 3(1): 15-29.

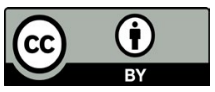

